\title{
Quantum statistics with internal symmetry
}

\author{
Hans-Thomas Elze and Walter Greiner \\ Institut für Theoretische Physik der Universität Frankfurt am Main, Postfach 111 932, D-6000 Frankfurt am Main, Germany \\ and Nuclear Science Division, Building 70A, Lawrence Berkeley Laboratory, University of California, \\ Berkeley, California 94720 \\ (Received 7 October 1985)
}

\begin{abstract}
We formulate a group-theoretical projection technique for the quantum-statistical description of systems with exactly conserved charges corresponding to local non-Abelian gauge symmetries. The formalism is specified for $S U(N)$ internal symmetry and a partition function related to a mixed canonical-grand-canonical ensemble is defined. Its perturbation expansion is derived, and we point out potential applications. We also study single-particle Green's functions for the calculation of mixed ensemble averages with the help of a generalized Wick's theorem and find that a connectedgraphs expansion is impossible.
\end{abstract}

\section{INTRODUCTION}

Quite often in physics we are studying many-particle systems with the help of statistical methods. Typically particle numbers are ranging from $10^{1}$ to $10^{23}$ and beyond, e.g., in elementary-particle, nuclear, or solid-state physics. All these systems, if sufficiently isolated from the rest of the world, obey the local conservation laws of energy, momentum, and angular momentum, reflecting invariance with respect to translations and rotations in space-time. But even for relativistic systems, where particle number conservation no longer holds, more locally conserved quantities can be identified, which are usually referred to as "charges" corresponding to local gauge symmetries. We will henceforth call them "internal symmetries" of a given theory. They can be distinguished from other "external" ones, e.g., discrete symmetries of a crystal, which arise due to energetically favored ground-state properties usually breaking some larger internal symmetry.

The purpose of our paper is to report a simple method to respect such internal symmetries in statistical descriptions of many-particle systems.

It amounts to the construction of suitable canonical ensembles to incorporate exactly the charge conservation for a given dynamical system. In the context of quantumstatistical mechanics our aim is to (implicitly) build Fock spaces of states which are characterized by definite symmetry properties or prescribed charges. A general projection technique to solve this problem for noninteracting systems was proposed by Redlich and Turko only a few years ago, ${ }^{1}$ but such considerations may be traced back to the famous work by Bethe, who calculated the energy and total spin dependence of the single-particle density of states in the nuclear Fermi-gas model. ${ }^{2}$ However, his approach was adapted to the particular SU(2) symmetry involved and could not be generalized. Three decades ago microcanonical calculations taking into account fourmomentum conservation ${ }^{3}$ and electric charge or baryonnumber conservation ${ }^{4}$ were carried out to describe particle production in inelastic nucleon-nucleon scattering at a few
$\mathrm{GeV}$ laboratory energy. More recently conservation of electriclike charges (baryon number, strangeness) was studied within the thermodynamical model of high-energy hadronic collisions ${ }^{5,6}$ and, among others, important finite-size effects were found-this will be a general feature to be met again and again whenever, in a statistical description, phase space or Fock space are restricted due to conservation laws.

It has been a common attribute of all applications of statistical methods to the study of charge-conserving systems mentioned so far (except Bethe's) that the underlying symmetry corresponded to one or several unrelated $U(1)$ groups. For a $U(1)$ symmetry, which means that electriclike charges of constituents can be simply added to obtain the total charge of a many-particle state, the technical treatment is rather simple. This was also noted in a field-theoretic formulation of the problem employing the functional integral representation of a partition function by Kapusta. ${ }^{7}$ Since the pioneering work of Yang and mills, ${ }^{8}$ however, there has been an ever increasing interest in gauge theories based on various non-Abelian internal symmetries. ${ }^{9}$ Therefore we would like to explain a group-theoretical method which allows us to treat nonAbelian symmetries rigorously in a canonical statistical description. In Sec. II we review the basic formalism and also show in detail how the results of Redlich and Turko can be extended to cover interacting systems. Furthermore, we introduce the concept of a mixed canonical-grand-canonical partition function, which will be useful under circumstances where some charges have to be conserved exactly while others need only be conserved on the average. Finally, we also consider in Sec. III single-particle Green's functions, which can be defined in connection with mixed canonical-grand-canonical ensembles. There we find important differences as compared with the usual finite-temperature perturbation theory.

For an illustration of the method and a detailed application the reader may be referred to previously reported phenomenological studies of an approximately noninteracting quark-gluon plasma. ${ }^{10-12}$ This fascinating new state of matter should be formed in ultrarelativistic nu- 
clear collisions of the present day or experiments in the near future, ${ }^{13}$ if one believes in the $\mathrm{SU}_{c}(3)$-color gauge theory of strong interactions [quantum chromodynamics (QCD)] formulated in terms of quarks and gluons. ${ }^{14}$ It has been demonstrated that the $\mathrm{SU}_{c}(3)$-singlet nature (colorlessness) of all physical (hadronic) states, the socalled confinement effect, has important consequences for thermodynamic properties of finite-size plasma droplets to be expected experimentally. ${ }^{10,11,15}$ Parts of the material presented in the following are contained in Ref. 15, but we also include here several new results, the general framework for perturbation theory in particular.

\section{PROJECTION TECHNIQUE FOR SU( $N)$ INTERNAL SYMMETRY GROUP}

To be definite, we restrict ourselves in the formal development of the method to the $\mathrm{SU}(N)$ group. As was stated in Ref. 1, however, and as will become clear in the following, it works for all compact semisimple Lie groups in the same manner-for the benefit of the reader, we recall some essential features of $\mathrm{SU}(N)$ in Appendix A, which also serves to define our notation. We choose units such that $\hbar=c=k_{B}=1$.

\section{A. Partition function}

Let $\hat{H}$ denote the Hamiltonian defining the dynamical system under investigation. Then we may define an exact internal $\mathrm{SU}(N)$ symmetry by the commutation relations

$\left[\hat{H}, \hat{g}_{\lambda}\right]=0=\left[\hat{H}, C_{v}\right], \lambda=1,2, \ldots, n, v=1,2, \ldots, r$

where $g_{\lambda}$ and $C_{v}$ are the $N^{2}-1=n$ generators and $N-1=r$ Casimir operators, respectively (see Appendix A). The second of Eqs. (1) is a consequence of the first one. Thus internal symmetry especially means vanishing commutators between the Hamiltonian and all generators of the Cartan subgroup and all Casimir operators. Together they form a set of $2(N-1)+1=2 N-1$ commuting operators which can be diagonalized simultaneously (in principle); their eigenvalues are often called the "good quantum numbers" of the physical states of the system.

After these preliminary remarks we state the aim of this section: It is the calculation of a canonical partition function $Z_{\mathrm{Q}}$ defined by

$$
Z_{\mathrm{Q}} \equiv \underset{\mathrm{Q}}{\operatorname{Tr}}\left(e^{-\beta \hat{H}}\right)
$$

where $\beta \equiv T^{-1}$ denotes the inverse temperature of the system and $V$ its volume; the usual trace of the statistical operator (density matrix) has to be taken under the essential constraint that all states involved must belong to an unitary irreducible representation of $S U(N)$ specified by the $(N-1)$-dimensional integer-valued vector $\mathbf{Q}$ (cf. Appendix $A$ ). The additional constraint that the physical Hilbert space considered is built up from (many-particle) states transforming as a definite multiplet with respect to the symmetry group makes it a canonical partition function, since the quantum numbers corresponding to $\mathbf{Q}$ are kept fixed. It can be understood as straightforward gen- eralization of the $U(1)$ case, where only states of a given total electriclike charge $Q$ would have to be taken into account in a canonical partition function $Z_{Q}$ (cf. Refs. 4-6). To get an impression of what is meant by $Z_{Q}$, it helps to think of SU(2) multiplets, where $Q$ is still one dimensional, a multiplet being specified, e.g., by an integer $2 J$ with $J$ the total spin quantum number.

Calculation of a partition function is a task central to all applications of quantum-statistical mechanics, therefore the description of systems with generalized nonAbelian charge conservation as expressed by Eqs. (1) and (2) together with Eqs. (A2) and (A4) is quite interesting in itself. However, as soon as the internal symmetry group is $\mathbf{S U}(2), \mathbf{S U}(3)$, or larger, the required construction of all many-particle states belonging to a given multiplet seems practically impossible; the situation looks even worse for relativistic systems without particle number conservation. Therefore, the projection technique suggested in Ref. 1 presents a major step forward. Its most important quantity turns out to be a new generating function $\widetilde{Z}$ defined by

$\widetilde{Z}\left(T, V ; \alpha_{1}, \alpha_{2}, \ldots, \alpha_{r}\right) \equiv \sum_{\mathbf{Q}} \frac{Z_{\mathbf{Q}}(T, V)}{\operatorname{dim}(\mathbf{Q})} \chi_{\mathbf{Q}}\left(\alpha_{1}, \alpha_{2}, \ldots, \alpha_{r}\right)$,

where a weighted summation is performed over all multiplets $\mathbf{Q}$ of canonical partition functions $Z_{\mathbf{Q}}$, the type one is ultimately looking for; the weights are characters $\chi_{Q}$ of the respective representations divided by their dimensions $\operatorname{dim}(\mathbf{Q})$ :

$$
\chi_{\mathbf{Q}}\left(\alpha_{1}, \alpha_{2}, \ldots, \alpha_{r}\right) \equiv \underset{\mathbf{Q}}{\operatorname{Tr}} \exp \left[i \sum_{\mu=1}^{r} \alpha_{\mu} \hat{Q}_{\mu}\right] .
$$

In this way the characters generally are complex-valued functions of those $r$ real variables $\alpha_{\mu}$ which parametrize elements of the Cartan subgroup, and using Eq. (4), we obtain the dimension of a representation from

$$
\operatorname{dim}(\mathbf{Q}) \equiv \operatorname{Tr}_{\mathbf{Q}} \mathbf{1}=\chi_{\mathbf{Q}}(0,0, \ldots, 0)
$$

The properties of group characters were analyzed by Weyl in the context of the theory of Lie group representations. ${ }^{16-18}$ We only borrow an orthogonality relation for group characters from these more general results valid for compact semisimple Lie groups:

$$
\begin{aligned}
\int_{\mathrm{SU}(N)} d \alpha_{1} d \alpha_{2} \cdots d \alpha_{r} M\left(\alpha_{1}, \alpha_{2}, \ldots, \alpha_{r}\right) \\
\quad \times \chi_{\mathrm{Q}^{*}}^{*}\left(\alpha_{1}, \alpha_{2}, \ldots, \alpha_{r}\right) \chi_{\mathrm{Q}^{\prime}}\left(\alpha_{1}, \alpha_{2}, \ldots, \alpha_{r}\right)=\delta_{\mathrm{QQ}^{\prime}},
\end{aligned}
$$

where the $r$-dimensional integration region is the parameter set of the Cartan subgroup and $M$ is a suitable weight function (Haar measure). Explicit realizations of Eqs. (4)-(6) for the cases of $U(1), S U(2)$, and $S U(3)$, which were (re)derived in Ref. 15, are compiled in Appendix B for easy reference.

By applying the orthogonality relation [Eq. (6)] to Eq. (3) we immediately find 


$$
Z_{\mathbf{Q}}(T, V)=\operatorname{dim}(\mathbf{Q}) \int_{\operatorname{SU}(N)} d \alpha_{1} d \alpha_{2} \cdots d \alpha_{r} M\left(\alpha_{1}, \alpha_{2}, \ldots, \alpha_{r}\right) \chi_{\mathbf{Q}}^{*}\left(\alpha_{1}, \alpha_{2}, \ldots, \alpha_{r}\right) \widetilde{Z}\left(T, V ; \alpha_{1}, \alpha_{2}, \ldots, \alpha_{r}\right),
$$

which formally solves the problem of finding the canonical partition function $Z_{\mathbf{Q}}$ by expressing it as a group integral over the generating function $\widetilde{Z}$, defined in Eqs. (2) and (3) respectively. Of course, nothing would be gained, could we not prove the following remarkable identity:

$\tilde{\mathbf{Z}}\left(T, V ; \alpha_{1}, \alpha_{2}, \ldots, \alpha_{r}\right)=\operatorname{Tr} \exp \left(-\beta \hat{H}+i \sum_{\mu=1}^{r} \alpha_{\mu} \hat{Q}_{\mu}\right)$,

which relates $\widetilde{Z}$ to an essentially grand-canonical "partition function," since now an unrestricted trace is allowed irrespective of any internal $S U(N)$ quantum numbers. It is not hard to imagine that the right-hand side (rhs) of Eq. (8) can be calculated in practice, which is not true for either Eq. (2) or (3). This is a most important result: While the canonical partition function $Z_{\mathrm{Q}}$ in general cannot be calculated directly, the unrestricted summation required for the generating function $\widetilde{Z}$ can be carried out. From the latter one can obtain the desired $Z_{\mathrm{Q}}$ in a rather straightforward way by exploiting relation (7), i.e., by projecting with a character $\chi_{\mathbf{Q}}$ of the symmetry group. In fact, for a noninteracting system all operators on the rhs of Eq. (8) can be expressed as linear combinations of number operators diagonal in Fock space; then $\widetilde{Z}$ can be calculated easily in complete analogy to free Fermi or Bose-gas partition functions, which is exemplified by our study of an idealized $\mathrm{SU}_{c}(3)$ quark-gluon plasma. ${ }^{10,11,15}$ For interacting systems with an exact internal symmetry we set up a perturbation theory in Sec. II B, which resembles the perturbative approach to the ordinary many-body problem.

To close this section, we prove Eq. (8) by calculating its rhs step by step. We begin by decomposing the Hilbert space according to irreducible unitary representations $\mathbf{Q}$ of $\operatorname{SU}(N)$ :

$$
\begin{aligned}
\operatorname{Tr} \exp & \left(-\beta \hat{H}+i \sum_{\mu=1}^{r} \alpha_{\mu} \hat{Q}_{\mu}\right) \\
& =\sum_{\mathbf{Q}} \operatorname{Tr}(\hat{\mathbf{\rho}} \hat{\gamma}) \\
& =\sum_{\mathbf{Q}} \sum_{n(\mathbf{Q})} \sum_{m(\mathbf{Q})}\langle n, m|\hat{\rho} \hat{\gamma}| n, m\rangle,
\end{aligned}
$$

where we introduced the statistical operator $\hat{\rho}$ (density matrix) and a general element of the Cartan subgroup $\hat{\gamma}$, respectively, defined by

$$
\hat{\rho} \equiv e^{-\beta \hat{H}}, \hat{\gamma} \equiv \exp \left(i \sum_{\mu=1}^{r} \alpha_{\mu} \hat{Q}_{\mu}\right) .
$$

The decomposition in Eq. $\left(8^{\prime}\right)$, where $n$ denotes members of a multiplet $\mathbf{Q}$ and $m$ accounts for additional degeneracies due to, e.g., varying particle content of a given multiplet ("principal quantum numbers"), is always possible (in principle), since we assumed exact internal symmetry as defined by Eqs. (1). Inserting a complete set of states, we obtain, for the rhs of Eq. ( $\left.8^{\prime}\right)$,

$$
\begin{aligned}
\sum_{\mathbf{Q}} \sum_{n, n^{\prime}} \sum_{m, m^{\prime}}\left\langle n, m|\hat{\rho}| n^{\prime}, m^{\prime}\right\rangle\left\langle n^{\prime}, m^{\prime}|\hat{\gamma}| n, m\right\rangle \\
=\sum_{\mathbf{Q}} \sum_{n} \sum_{m}\langle n, m|\hat{\rho}| n, m\rangle\langle n|\hat{\gamma}| n\rangle ;
\end{aligned}
$$

in the last equality use was made of the fact that $\hat{\rho}$ as well as $\hat{H}$ can be chosen diagonal with respect to a representation $\mathbf{Q}$ because of Eqs. (1) and that $\hat{\gamma}$ is diagonal with respect to the substates $m$, since the charge operators $\hat{Q}_{\mu}$ only act on states $n$ of a multiplet [ $n$ corresponds to the magnetic quantum number in the case of SU(2) symmetry]. Furthermore, because of Eqs. (1) there is no finestructure splitting for an exact internal symmetry and matrix elements of $\hat{H}$ are constant within a given multiplet (i.e., independent of $n$ ), which implies that the right-hand side of $\left(8^{\prime}\right)$ is also

$$
\begin{aligned}
\sum_{\mathbf{Q}} \sum_{m(\mathbf{Q})}\langle m|\hat{\rho}| m\rangle \sum_{n(\mathbf{Q})}\langle n|\hat{\gamma}| n\rangle \\
=\sum_{\mathbf{Q}} \frac{\sum_{n(\mathbf{Q})}}{\operatorname{dim}(\mathbf{Q})} \sum_{m(\mathbf{Q})}\langle m|\hat{\rho}| m\rangle \chi_{\mathbf{Q}} \\
=\sum_{\mathbf{Q}} \frac{Z_{\mathbf{Q}}}{\operatorname{dim}(\mathbf{Q})} \chi_{\mathbf{Q}}, \quad\left(8^{\prime \prime \prime}\right)
\end{aligned}
$$

where we used the definitions of Eqs. (9), (4), and (2) in the second and last equality, respectively; by the definition of the generating function $\widetilde{Z}$ in Eq. (3) this completes the proof of Eq. (8).

\section{B. Perturbation theory}

for mixed canonical-grand-canonical partition function

Our development of perturbation theory is based on the observation that in deriving the projection formalism in order to respect exact internal symmetry in Sec. II A, nowhere did the assumption of a noninteracting manyparticle system enter. Therefore, the main results obtained there, which can be summarized by Eqs. (4), (7), and (8), are valid for interacting systems too. ${ }^{15}$ However, a genuinely nondiagonal Hamiltonian present through the density matrix, Eq. (9), on the rhs of Eq. (8), which has to be calculated in any practical application, generally is hard to deal with, which suggests a perturbative expansion of the generating function $\bar{Z}$.

Before we start with perturbation theory, we introduce as a slight generalization of the canonical partition function defined in Eq. (2) a "mixed canonical-grandcanonical" partition function:

$$
\begin{aligned}
Z_{\mathbf{Q}}\left(T, V, \mu_{1}, \mu_{2}, \ldots, \mu_{m}\right) & \\
& \equiv \underset{\mathbf{Q}}{\operatorname{Tr} \exp }\left[-\beta\left(\hat{H}-\sum_{k=1}^{m} \mu_{k} \hat{q}_{k}\right)\right],
\end{aligned}
$$

i.e., we define a canonical partition function with respect to an exact internal $\mathrm{SU}(N)$ symmetry with multiplets $\mathrm{Q}$ 
as in Sec. II A; but the partition function given in Eq. (10) also allows for an average (grand-canonical) conservation of $m$ additive charges described by linear operators $\hat{q}_{k}$ via chemical potentials $\mu_{k}$ as usual. The formal derivation and results obtained in the previous section remain valid, provided the charge generators $\widehat{q}_{k}$ external to the set of $\mathrm{SU}(N)$ generators $\hat{g}_{\lambda}$ do not break the exact internal symmetry, which means that Eqs. (1) should be complemented by

$$
\left[\hat{q}_{k}, \hat{g}_{\lambda}\right]=0, k=1,2, \ldots, m, \lambda=1,2, \ldots, n ;
$$

as in Eqs. (1) this implies

$$
\left[\hat{q}_{k}, \widehat{C}_{v}\right]=0, k=1,2, \ldots, m, v=1,2, \ldots, r .
$$

Of course, the replacement

$$
\hat{H} \rightarrow \hat{H}-\sum_{k=1}^{m} \mu_{k} \hat{q}_{k} \equiv \hat{H}(\boldsymbol{\mu})
$$

has to be made consistently throughout Sec. II A. We could include the terms involving chemical potentials in the definition of a new Hamiltonian $\hat{H}\left(\mu_{1}, \mu_{2}, \ldots, \mu_{m}\right)$ depending on external parameters $\mu_{k}$; because of Eq. (1') $\hat{H}(\boldsymbol{\mu})[$ Eq. (11)] satisfies Eqs. (1) and the formalism works as before. We notice that a group structure to which the operators $\hat{q}_{k}$ could belong must not necessarily be known for the derivation of the following equation replacing Eq. (8):

$$
\begin{aligned}
\tilde{Z}\left(T, V, \mu_{1}, \mu_{2}, \ldots, \mu_{m} ; \alpha_{1}, \alpha_{2}, \ldots, \alpha_{r}\right) \\
=\operatorname{Tr} \exp \left[-\beta\left[\hat{H}-\sum_{k=1}^{m} \mu_{k} \hat{q}_{k}\right]+i \sum_{\mu=1}^{r} \alpha_{\mu} \hat{Q}_{\mu}\right] ;
\end{aligned}
$$

$Z_{\mathbf{Q}}$ [Eq. (10)] again is obtained by operating on $\widetilde{Z}$ [Eq. (12)] with the projection and integration as in Eq. (7).

Finally, we remark that the consideration of a mixed canonical-grand-canonical partition function to us seems worthwhile because of potential applications as already discussed in the quark-gluon plasma case; ${ }^{11,15}$ generally speaking, there are physical situations where a finite sys- tem easily exchanges some charges or quantum numbers with a surrounding (heat) bath, while others are strictly confined to or excluded from it. Other generalizations, e.g., for exact $\mathrm{SU}(N) \times \mathrm{SU}(M)$ internal symmetry, could be worked out as well.

For the derivation of a perturbative expansion of the generating function $\widetilde{Z}$, Eq. (12), we split the Hamiltonian together with the terms depending on chemical potentials into two parts:

$$
\hat{H}(\boldsymbol{\mu}) \equiv \hat{H}_{0}-\sum_{k=1}^{m} \mu_{k} \hat{q}_{k}+\hat{H}_{1} \equiv \hat{H}_{0}(\boldsymbol{\mu})+\hat{H}_{1},
$$

where $\hat{H}_{0}$ is chosen such that it can be easily diagonalized, e.g., in Fock space, and $\hat{H}_{1}$ describes some perturbing interaction. Furthermore, we require that $\hat{H}_{0}$ itself also commute with all generators of internal symmetry:

$$
\left[\hat{H}_{0}, \hat{g}_{\lambda}\right]=0, \lambda=1,2, \ldots, n,
$$

which implies by Eqs. (1), (1'), and (13)

$$
\left[\hat{H}_{0}(\boldsymbol{\mu}), \hat{g}_{\lambda}\right]=\left[\hat{H}_{1}, \hat{g}_{\lambda}\right]=0, \lambda=1,2, \ldots, n \text {. }
$$

Considering in the following density matrices $\hat{\rho}^{\mu}$ and $\hat{\rho}_{0}^{\mu}$, respectively, defined by

$$
\begin{aligned}
& \hat{\rho}^{\boldsymbol{\mu}} \equiv e^{-\beta\left[\hat{H}_{0}(\mu)+\hat{H}_{1}\right]}, \\
& \hat{\rho}_{0}^{\boldsymbol{\mu}} \equiv e^{-\beta \hat{H}_{0}(\boldsymbol{\mu})},
\end{aligned}
$$

we obtain an operator differential equation to be satisfied by $\hat{\rho}^{\mu}$ :

$$
\frac{\partial \hat{\rho}^{\mu}}{\partial \beta}=-\left[\hat{H}_{0}(\mu)+\hat{H}_{1}\right] \hat{\rho}^{\mu},
$$

together with the "initial condition"

$$
\hat{\rho}^{\mu}(\beta=0)=1 \text {. }
$$

Equations (16) are well known and can be formally solved by iteratively solving an equivalent integral equation. ${ }^{21,22}$ One starts with $\hat{\rho}_{0}^{\mu}$ as the zeroth approximation and finally obtains

$$
\hat{\rho}^{\mu}=\sum_{n=0}^{\infty} \frac{(-1)^{n}}{n !} \int_{0}^{\beta} d \beta_{1} \int_{0}^{\beta} d \beta_{2} \cdots \int_{0}^{\beta} d \beta_{n} \hat{\rho}_{0}^{\mu} T_{\beta}\left[H_{1}\left(\beta_{1}\right) H_{1}\left(\beta_{2}\right) \cdots H_{1}\left(\beta_{n}\right)\right]
$$

where $T_{\beta}$ denotes the "time-ordering" operator placing the interaction at the latest "time" $\beta_{i}$ farthest to the left, the one at the next latest time next, etc., and where the "interaction-picture" operators are defined by

$$
\hat{H}_{1}(\tau) \equiv e^{\tau \hat{H}_{0}(\mu)} \hat{H}_{1} e^{-\tau \hat{H}_{0}(\mu)}
$$

Naturally the perturbative expansion, Eq. (17), is rather similar to the Dyson expansion of the $S$ matrix in field theory, because Eq. (16a) represents an "imaginary-time" Schrödinger equation.

Inserting this result into Eq. (12), we find the wanted expansion for the generalized generating function $\widetilde{Z}$ :

$$
\widetilde{Z}(T, V, \mu ; \boldsymbol{\alpha})=\sum_{n=0}^{\infty} \frac{(-1)^{n}}{n !} \int_{0}^{\beta} d \beta_{1} \int_{0}^{\beta} d \beta_{2} \cdots \int_{0}^{\beta} d \beta_{n} \operatorname{Tr}\left\{\hat{\rho}_{0}^{\mu} T_{\beta}\left[\hat{H}_{1}\left(\beta_{1}\right) \hat{H}_{1}\left(\beta_{2}\right) \cdots \hat{H}_{1}\left(\beta_{n}\right)\right] \hat{\gamma}(\boldsymbol{\alpha})\right\}
$$

with $\hat{\gamma}$ an element of the internal Cartan subgroup as defined in the second of Eqs. (9). Since $\hat{\gamma}$ commutes with all 
operators in front of it on the rhs of Eq. (19) on account of Eqs. (14) and (15), we may rewrite the argument of the trace:

$$
\begin{aligned}
\hat{\rho}_{0}^{\mu} T_{\boldsymbol{\beta}}\left[\hat{H}_{1}\left(\beta_{1}\right) \hat{H}_{1}\left(\beta_{2}\right) \cdots \hat{H}_{1}\left(\beta_{n}\right)\right] & \hat{\gamma}(\boldsymbol{\alpha}) \\
& =e^{-\boldsymbol{\beta} \hat{H}_{0}(\boldsymbol{\mu}, \boldsymbol{\alpha})} T_{\boldsymbol{\beta}}\left(e^{\boldsymbol{\beta}_{1} \hat{H}_{0}(\boldsymbol{\mu}, \boldsymbol{\alpha})} \hat{H}_{1} e^{-\beta_{1} \hat{H}_{0}(\boldsymbol{\mu}, \boldsymbol{\alpha})} e^{\boldsymbol{\beta}_{2} \hat{H}_{0}(\boldsymbol{\mu}, \boldsymbol{\alpha})} \hat{H}_{1} e^{-\beta_{2} \hat{H}_{0}(\boldsymbol{\mu}, \boldsymbol{\alpha})} \cdots e^{\boldsymbol{\beta}_{n} \hat{H}_{0}(\boldsymbol{\mu}, \boldsymbol{\alpha})} \hat{H}_{1} e^{-\beta_{n} \hat{H}_{0}(\boldsymbol{\mu}, \boldsymbol{\alpha})}\right),
\end{aligned}
$$

where we used Eq. (18), reexpressed $\hat{\gamma}$ in terms of generators, and made use of the abbreviation [cf. Eq. (13)]

$$
\hat{H}_{0}(\boldsymbol{\mu}, \boldsymbol{\alpha}) \equiv \hat{H}_{0}(\boldsymbol{\mu})-\frac{i}{\beta} \sum_{\mu=1}^{r} \alpha_{\mu} \hat{Q}_{\mu}
$$

Inserting Eq. (20) together with Eq. (21) into Eq. (19), we observe that the perturbative expansion of the generating function $\widetilde{Z}$ becomes identical to the known expansion of the grand canonical partition function, ${ }^{22}$ where now, however, some "chemical potentials" corresponding to $i \alpha_{1} / \beta, \ldots, i \alpha_{r} / \beta$ are purely imaginary.

Combining the results obtained in this section so far with the general formalism of Sec. II A, we may summarize the projection method for a mixed canonical-grand-canonical partition function by the formulas

$$
\begin{aligned}
& Z_{\mathbf{Q}}(T, V, \boldsymbol{\mu})=\operatorname{dim}(\mathbf{Q}) \int_{\mathrm{SU}(N)} d \alpha_{1} d \alpha_{2} \cdots d \alpha_{r} M(\boldsymbol{\alpha}) \chi_{\mathbf{Q}}^{*}(\boldsymbol{\alpha}) \widetilde{\boldsymbol{Z}}(T, V, \boldsymbol{\mu} ; \boldsymbol{\alpha}), \\
& \widetilde{\boldsymbol{Z}}(T, V, \boldsymbol{\mu} ; \boldsymbol{\alpha})=\sum_{n=0}^{\infty} \frac{(-1)^{n}}{n !} \int_{0}^{\beta} d \beta_{1} \int_{0}^{\beta} d \beta_{2} \cdots \int_{0}^{\beta} d \beta_{n} \operatorname{Tr}\left[e ^ { - \beta \hat { H } _ { 0 } ( \boldsymbol { \mu } , \boldsymbol { \alpha } ) } T _ { \beta } \left(e^{\beta_{1} \hat{H}_{0}(\boldsymbol{\mu}, \boldsymbol{\alpha})} \hat{H}_{1} e^{-\beta_{1} \hat{H}_{0}(\boldsymbol{\mu}, \boldsymbol{\alpha})}\right.\right. \\
&\left.\left.\times \cdots e^{\beta_{n} \hat{H}_{0}(\boldsymbol{\mu}, \boldsymbol{\alpha})} \hat{H}_{1} e^{-\beta_{n} \hat{H}_{0}(\boldsymbol{\mu}, \boldsymbol{\alpha})}\right)\right],
\end{aligned}
$$

where $\chi_{\mathbf{Q}}(\boldsymbol{\alpha})$ and $\hat{H}_{0}(\boldsymbol{\mu}, \boldsymbol{\alpha})$ are defined by Eqs. (4) and (21), respectively. It is very important to remark here that the calculation of perturbative corrections for the generating function $\widetilde{Z}$ as given by Eq. (22b) can be performed by applying Wick's theorem in its usual finite-temperature form, ${ }^{22}$ however, including the modification of complex "chemical potentials" as discussed in connection with Eq. (21). The derivation of Wick's theorem, which relates (noninteracting) grand-canonical ensemble averages of time-ordered products of field operators, does not depend on the chemical potentials necessarily being real numbers. Therefore well-known results concerning grand-canonical partition functions of many-particle systems, whose interactions are treated perturbatively, can immediately be generalized to respect exact internal symmetries. This observation opens the way to interesting new applications of the projection formalism. Droplets of weakly interacting quark-gluon plasma are presently under investigation.

\section{SINGLE-PARTICLE TEMPERATURE GREEN'S FUNCTIONS}

The study of single-particle temperature Green's functions is motivated by the fact that they can be related to equilibrium thermodynamic properties of the system, i.e., ensemble averages of general one-body operators and of the two-body interaction energy in particular. ${ }^{22,23}$ The purpose of this section is to define and investigate singleparticle temperature Green's functions appropriate, e.g., for the calculation of mixed canonical-grand-canonical ensemble averages. We will call them $m$-Green's functions henceforth. Our presentation parallels the considerations in Ref. 22, where the Green's functions related to grand-canonical ensemble averages are studied, which we call $g$-Green's functions in the following.

Using the mixed canonical-grand-canonical partition function $Z_{\mathrm{Q}}$ and the density matrix $\hat{\rho}^{\mu}$, respectively, defined in Eqs. (10) and (15) in Sec. II B, we define the $m$ Green's function $G_{\mathrm{Q}}$ :

$$
\begin{aligned}
& G_{\mathrm{Q}}\left(j, \tau ; j^{\prime}, \tau^{\prime}\right) \\
& \quad \equiv-Z_{\mathrm{Q}}^{-1} \underset{\mathrm{Q}}{\operatorname{Tr}}\left(\hat{\rho}^{\mu} T_{\tau}\left\{\left[\hat{a}_{j}(\tau)\right]_{H}\left[\hat{a}_{j}^{\dagger},\left(\tau^{\prime}\right)\right]_{H}\right\}\right),
\end{aligned}
$$

with the "Heisenberg-picture" operators,

$$
\left[\hat{a}_{j}^{(\dagger)}(\tau)\right]_{H} \equiv e^{\tau \hat{H}(\boldsymbol{\mu})} \hat{a}_{j}^{(\dagger)} e^{-\tau \hat{H}(\boldsymbol{\mu})}
$$

$j$ denotes a complete set of single-particle space-time and internal quantum numbers and $\hat{a}_{j}^{+}, \hat{a}_{j}$ are the corresponding creation and destruction operators satisfying (anti)commutation rules for bosons (fermions). The timeordering operator $T_{\tau}$ is defined here to include a factor $(-1)^{P}$ for fermions, where $P$ is the number of permutations of fermion operators required to perform time ordering, which was introduced previously [cf. Eq. (17)]. We do not explicitly distinguish between particles and antiparticles and suppressed the dependence on thermodynamic variables $T, V, \mu$ in $G_{\mathrm{Q}}$ in order to keep the notation as simple as possible. Of course, the definition given in Eq. (23) resembles the one of an ordinary g-Green's function $G$ :

$$
G\left(j, \tau ; j^{\prime}, \tau^{\prime}\right) \equiv-Z^{-1} \operatorname{Tr}\left(\hat{\rho}^{\mu} T_{\tau}\left\{\left[\hat{a}_{j}(\tau)\right]_{H}\left[\hat{a}_{j^{\prime}}^{\dagger}\left(\tau^{\prime}\right)\right]_{H}\right\}\right),
$$

where $Z$ is the usual grand-canonical partition function.

We immediately conclude that $G_{\mathrm{Q}}$ and $G$ share the same analytical properties, as far as they only depend on 
the operator structures following the trace symbols in Eqs. (23) and (25): 22,23

$$
\boldsymbol{G}_{(\mathbf{Q})}\left(j, \tau ; j^{\prime}, \tau^{\prime}\right)=G_{(\mathbf{Q})}\left(j, j^{\prime} ; \tau-\tau^{\prime}\right),
$$

i.e., $G_{(Q)}$ depends only on the difference of the "time" arguments, which follows from the Hamiltonian being independent of temperature; furthermore, it is necessary and sufficient for convergence of the traces that $-\beta$ $<\tau-\tau^{\prime}<\beta .^{23}$ Then one obtains from Eq. (23) or Eq. (25) together with Eq. (24), for $0<\tau<\tau^{\prime}<\beta$,

$$
\begin{aligned}
\boldsymbol{G}_{(\mathbf{Q})}\left(j, j^{\prime} ; \tau-\tau^{\prime}\right) & = \pm Z_{(\mathbf{Q})}^{-1} \underset{(\mathbf{Q})}{\operatorname{Tr}}\left\{\hat{\rho}^{\mu}\left[\hat{a}_{j^{\prime}}^{\dagger}\left(\tau^{\prime}\right)\right]_{H}\left[\hat{a}_{j}(\tau)\right]_{H}\right\} \\
& = \pm Z_{(\mathbf{Q})}^{-1} \operatorname{Tr}_{(\mathbf{Q})}\left\{\left[\hat{a}_{j}(\tau)\right]_{H} e^{-\beta \hat{H}(\boldsymbol{\mu})}\left[\hat{a}_{j^{\prime}}^{\dagger}\left(\tau^{\prime}\right)\right]_{H}\right\} \\
& = \pm Z_{(\mathbf{Q})}^{-1} \operatorname{Tr}_{(\mathbf{Q})}\left\{\hat{\rho}^{\mu}\left[\hat{a}_{j}(\tau+\beta)\right]_{H}\left[\hat{a}_{j^{\prime}}^{\dagger}\left(\tau^{\prime}\right)\right]_{H}\right\} \\
& =\mp G_{(\mathbf{Q})}\left(j, j^{\prime} ; \tau-\tau^{\prime}+\beta\right),
\end{aligned}
$$

where use was made of a cyclic permutation of operators under the trace, which does not change its value; similarly, for $0<\tau^{\prime}<\tau<\beta$,

$$
\boldsymbol{G}_{(\mathbf{Q})}\left(j, j^{\prime} ; \tau-\tau^{\prime}\right)=\mp G_{(\mathbf{Q})}\left(j, j^{\prime} ; \tau-\tau^{\prime}-\beta\right),
$$

where the upper (lower) sign refers to fermions (bosons). It is well known that the periodicity properties, Eqs. (26b) and (26c), lead to considerable simplifications ("Matsubara frequency representation"), if Feynman rules for the perturbative evaluation of Green's functions are ultimately formulated in momentum space; ${ }^{22,23}$ however, we do not pursue this point and presently consider perturbation theory in coordinate space at first.

To find a perturbation expansion of the $m$-Green's function in terms of a perturbing interaction $\hat{H}_{1}$ [cf. Eq. (13)], it is easiest to copy the path which is usually followed, when dealing with $g$-Green's functions: ${ }^{22}$ It consists of a transcription of Eq. (23) to the interaction picture [cf. Eq. (18)] and subsequent insertion of a perturbation expansion for the time-development operator

$$
\hat{U}\left(\tau, \tau^{\prime}\right) \equiv \exp \left(\tau \hat{H}_{0}\right) \exp \left[-\left(\tau-\tau^{\prime}\right) \hat{H}\right] \exp \left(-\tau^{\prime} \hat{H}_{0}\right),
$$

which resembles Eq. (17) in Sec. II B and is obtained in a similar way. Nowhere does the restriction of the trace in Eq. (23) defining $G_{Q}$ to a definite unitary irreducible representation $\mathbf{Q}$ of $S U(N)$ present an additional complication, since the perturbation expansion is solely based on operator properties. The restricted trace still has to be performed in the intermediate result:

$$
\begin{aligned}
& G_{\mathbf{Q}}\left(j, j^{\prime} ; \tau-\tau^{\prime}\right) \\
& \quad=-Z_{\mathbf{Q}}^{-1} \operatorname{Tr}_{\mathbf{Q}}\left(\hat{\rho}_{0}^{\mu} \sum_{n=0}^{\infty} \frac{(-1)^{n}}{n !} \int_{0}^{\beta} d \tau_{1} \int_{0}^{\beta} d \tau_{2} \cdots \int_{0}^{\beta} d \tau_{n} T_{\tau}\left[\hat{H}_{1}\left(\tau_{1}\right) \hat{H}_{1}\left(\tau_{2}\right) \cdots \hat{H}_{1}\left(\tau_{n}\right) \widehat{a}_{j}(\tau) \hat{a}_{j^{\prime}}^{\dagger}\left(\tau^{\prime}\right)\right]\right),
\end{aligned}
$$

where $\hat{\rho}_{0}^{\mu}$ denotes the no-interaction density matrix, cf. Eqs. (15), and interaction-picture operators $\hat{H}_{1}(\tau), \hat{a}^{(\dagger)}(\tau)$ follow from the Heisenberg-picture ones by turning off the interaction [cf. Eqs. (18) and (24)]. Rewriting $Z_{Q}$ [Eq. (10)] in the denominator of Eq. (27) by exploiting the perturbation expansion of the density matrix $\hat{\rho}^{\mu}$ [Eq. (17)], we find

$$
G_{\mathbf{Q}}\left(j, j^{\prime} ; \tau-\tau^{\prime}\right)=-\frac{\sum_{n=0}^{\infty} \frac{(-1)^{n}}{n !} \int_{0}^{\beta} d \tau_{1} \int_{0}^{\beta} d \tau_{2} \cdots \int_{0}^{\beta} d \tau_{n} Z_{\mathbf{Q}^{0}}^{-1} \operatorname{Tr}\left\{\hat{\rho}_{0}^{\mu} T_{\tau}\left[\hat{H}_{1}\left(\tau_{1}\right) \hat{H}_{1}\left(\tau_{2}\right) \cdots \hat{H}_{1}\left(\tau_{n}\right) \hat{a}_{j}(\tau) \hat{a}_{j^{\prime}}^{\dagger}\left(\tau^{\prime}\right)\right]\right\}}{\sum_{n=0}^{\infty} \frac{(-1)^{n}}{n !} \int_{0}^{\beta} d \tau_{1} \int_{0}^{\beta} d \tau_{2} \cdots \int_{0}^{\beta} d \tau_{n} Z_{\mathbf{Q}^{0}}^{-1} \underset{\mathbf{Q}}{\operatorname{Tr}}\left\{\hat{\rho}_{0}^{\mu} T_{\tau}\left[\hat{H}_{1}\left(\tau_{1}\right) \hat{H}_{1}\left(\tau_{2}\right) \cdots \hat{H}_{1}\left(\tau_{n}\right)\right]\right\}}
$$

the no-interaction mixed canonical-grand-canonical partition function $Z_{Q^{0}}$ is multiplied into numerator and denominator for later convenience. As could be expected, the resulting expression for $G_{Q}$, Eq. (28), still has an appearance similar to the usual one for a $g$-Green's function $^{22}$ with the exception of subscripts $Q$, which make the important (physical) difference.

We observe that for the evaluation of a general term in Eq. (28) we necessarily need to calculate (noninteracting) ensemble averages of the following type:

$$
\begin{aligned}
Z_{\mathbf{Q}^{0}}^{-1} \operatorname{Tr}\left\{\hat{\rho}_{0}^{\mu} T_{\tau}\left[\hat{A}\left(\tau_{A}\right) \hat{B}\left(\tau_{B}\right) \hat{C}\left(\tau_{C}\right) \cdots \hat{X}\left(\tau_{X}\right)\right]\right\} \\
\equiv\left\langle T_{\tau}[\hat{A} \hat{B} \hat{C} \cdots \hat{X}]\right\rangle_{\mathbf{Q}^{0}},
\end{aligned}
$$

where $\hat{A}, \hat{B}, \hat{C}, \ldots, \hat{X}$ are field operators or creation and destruction operators in the interaction picture, since we assume the interaction $\hat{H}_{1}$ at least to consist of a pair of field operators,

$$
\hat{H}_{1} \equiv \int d^{3} x \hat{\psi}^{\dagger}(x) \hat{h}_{1} \hat{\psi}(x) .
$$

$\hat{h}_{1}$, e.g., may contain an operator in the space of internal quantum numbers and a function in coordinate space (external potential), another pair of field operators (selfcoupled field), or a second kind of field (minimally coupled fields). The field operators can be expanded,

$$
\hat{\psi}^{(\dagger)}(x)=\sum_{j} \phi_{j}^{(*)}(x) \hat{a}_{j}^{(\dagger)},
$$

with the complete set of eigenfunctions $\phi_{j}$ satisfying a modified time-independent Schrödinger equation:

$$
\hat{H}_{0}(\boldsymbol{\mu}) \phi_{j}(x)=e_{j}(\boldsymbol{\mu}) \phi_{j}(x) ;
$$

$e_{j}(\boldsymbol{\mu}) \equiv e_{j}-\boldsymbol{\mu} \cdot \mathbf{c}_{j}$, where $e_{j}$ denotes the ordinary eigenvalue for $\boldsymbol{\mu}=\mathbf{0}$ and $\boldsymbol{c}_{j}$ is the vector of (grand canonically treat- 
ed) charges belonging to a single-particle state, completely specified by $j$.

Now we define two types of contractions, an $m$ contraction,

$$
\widehat{A} \sim \hat{B}^{\sim} \equiv Z_{\mathrm{Q}^{0}}^{-1} \operatorname{Tr}\left(\hat{\rho}_{0}^{\mu} T_{\tau}[\hat{A} \hat{B}]\right) \equiv\left\langle T_{\tau}[\hat{A} \hat{B}]\right\rangle_{\mathrm{Q}^{0}},
$$

which thus is related to a (noninteracting) mixed ensemble average, and a $g$-contraction related to the (noninteracting) grand-canonical ensemble,

$$
\widehat{A} \cdot \hat{B} \cdot \equiv Z_{0}^{-1} \operatorname{Tr}\left(\hat{\rho}_{0}^{\mu} T_{\tau}[\hat{A} \hat{B}]\right) \equiv\left\langle T_{\tau}[\hat{A} \hat{B}]\right\rangle_{0} .
$$

With the help of the following generalized Wick's theorem it will be possible to evaluate expressions of the type required by the perturbation expansion, Eq. (28), or the rhs of Eq. (29) in terms of sums of fully contracted terms which are calculable in practice, as will be shown in a moment. In Appendix $\mathrm{C}$ we prove the generalization of Wick's theorem at finite temperature respecting exact internal symmetry:

$$
\begin{aligned}
& \left\langle T_{\tau}[\hat{A} \hat{B} \widehat{C} \cdots \hat{M} \cdots \hat{N} \cdots \hat{X}]\right\rangle_{\mathrm{Q}^{0}} \\
& =\hat{A}^{\prime} \cdot \hat{B} \cdot \hat{C}^{\cdots} \cdots \hat{M}^{\sim} \cdot \hat{N}^{\sim} \cdot \hat{X}^{\cdots}
\end{aligned}
$$

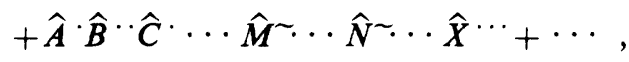

i.e., the mixed canonical-grand-canonical (noninteracting) ensemble average of products of interaction-picture field operators or creation and destruction operators is equal to the sum of all possible fully contracted terms, with $[\hat{A} \cdot \hat{B} \cdot \hat{C} \cdot \cdots] \equiv(\mp)[\hat{A} \cdot \hat{C} \cdot \hat{B} \cdots \cdots]$, etc., for fermions (bosons); however, each term on the rhs contains exactly one $m$ contraction, which essentially differs from the grand-canonical case, where only $g$-contractions occur. $^{22}$

To be able to apply Wick's theorem, Eq. (32), most economically, it is useful to relate the contractions defined in Eqs. (31) to free $g$ - and $m$-Green's functions. For simplicity we only consider contractions between creation and destruction operators (cf. Appendix C); to extend the results to field operators, one has to carry out the summations over normal modes of the fields as implied by Eq. (30b). We obtain directly from the definitions of the $\mathrm{m}$ Green's function, Eq. (23) [cf. Eq. (26a)], for the noninteracting ensemble and of the $m$ contraction, Eq. (31a):

$$
\left.G_{\mathbf{Q}}^{0}\left(j, j^{\prime} ; \tau-\tau^{\prime}\right)=-\left[\hat{a}_{j}(\tau)\right] \sim \hat{a}_{j^{\prime}}^{\dagger}\left(\tau^{\prime}\right)\right] \sim
$$

furthermore, by Eq. (31a) and Eq. (23) again,

$$
\begin{aligned}
{\left.\left[\hat{a}_{j}^{\dagger}(\tau)\right] \tau \hat{a}_{j^{\prime}}\left(\tau^{\prime}\right)\right]^{\sim} } & =\left\{\begin{array}{l}
\left\langle\hat{a}_{j}^{\dagger}(\tau) \hat{a}_{j^{\prime}}\left(\tau^{\prime}\right)\right\rangle \mathbf{Q}^{0}, \quad \tau>\tau^{\prime} \\
\mp\left\langle\hat{a}_{j^{\prime}}\left(\tau^{\prime}\right) \hat{a}_{j}^{\dagger}(\tau)\right\rangle \mathbf{Q}^{0}, \quad \tau^{\prime}>\tau
\end{array}\right. \\
& =\mp\left\langle T_{\tau}\left[\hat{a}_{j^{\prime}}\left(\tau^{\prime}\right) \hat{a}_{j}^{\dagger}(\tau)\right]\right\rangle_{\mathbf{Q}^{0}} \\
& = \pm G_{\mathbf{Q}^{\prime}}^{0}\left(j^{\prime}, j ; \tau^{\prime}-\tau\right) .
\end{aligned}
$$

Similarly,

$$
\begin{aligned}
& G^{0}\left(j, j^{\prime} ; \tau-\tau^{\prime}\right)=-\left[\hat{a}_{j}(\tau)\right]^{\cdot}\left[\hat{a}_{j^{\prime}}^{\dagger}\left(\tau^{\prime}\right)\right]^{\prime}, \\
& G^{0}\left(j^{\prime}, j ; \tau^{\prime}-\tau\right)= \pm\left[\hat{a}_{j}^{\dagger}(\tau)\right]^{\cdot}\left[\hat{a}_{j^{\prime}}\left(\tau^{\prime}\right)\right]^{\prime},
\end{aligned}
$$

cf. Eqs. (25) and (31b). Therefore, all kinds of possible contractions can be expressed by either a $g$ - or $m$-Green's function evaluated in the noninteracting ensemble.

The $g$-Green's function $G^{0}$ can be further evaluated with the help of Eqs. (C8) and (C9) derived in Appendix C and Eqs. (33c) and (33d):

$$
G^{0}\left(j, j^{\prime} ; \tau-\tau^{\prime}\right)=-\delta_{j j^{\prime}}\left(1 \mp\left\langle\hat{a}_{j}^{\dagger} \hat{a}_{j}\right\rangle_{0}\right) e^{-e_{j}(\mu)\left(\tau-\tau^{\prime}\right)}
$$

for $\tau>\tau^{\prime}$, and

$$
\begin{aligned}
G^{0}\left(j, j^{\prime} ; \tau-\tau^{\prime}\right) & = \pm\left\langle T_{\tau}\left[\hat{a}_{j^{\prime}}^{\dagger}\left(\tau^{\prime}\right) a_{j}(\tau)\right]\right\rangle_{0} \\
& = \pm \delta_{j j^{\prime}}\left\langle\hat{a}_{j}^{\dagger} \hat{a}_{j}\right\rangle_{0} e^{-e_{j}(\boldsymbol{\mu})\left(\tau-\tau^{\prime}\right)}
\end{aligned}
$$

for $\tau^{\prime}>\tau$. Concerning the free $m$-Green's function $G_{Q}^{0}$ we obtain directly from Eq. (23), using Eqs. (C3a) and (C3b) again,

$$
\begin{aligned}
& G_{\mathrm{Q}^{\prime}}^{0}\left(j, j^{\prime} ; \tau-\tau^{\prime}\right)=-Z_{\mathrm{Q}^{0}}^{-1} \underset{\mathrm{Q}}{\operatorname{Tr}}\left\{\hat{\rho}_{0}^{\mu} T_{\tau}\left[\hat{a}_{j}(\tau) \hat{a}_{j^{\prime}}^{\dagger}\left(\tau^{\prime}\right)\right]\right\}
\end{aligned}
$$

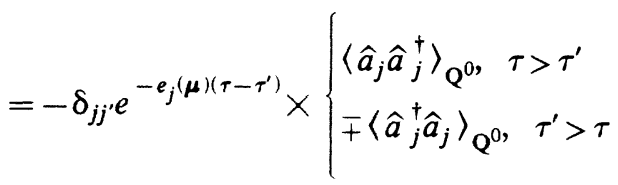

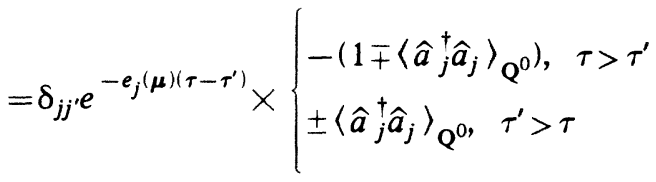

where in the second equality we respected the fact that states of the noninteracting mixed canonical-grandcanonical ensemble form a subset of all states defining the diagonal representation of $\hat{H}_{0}$; therefore only the above operator combinations diagonal in single-particle quantum numbers $j$ can have nonvanishing expectation values; in the last equality, of course, we applied the (anti)commutation rules. Now, what is the mixed canonical-grand-canonical ensemble average of a number operator, $\left\langle\hat{a}_{j}^{\dagger} \hat{a}_{j}\right\rangle_{\mathbf{Q}^{0}}$ ? By following the same line of argument which led to projection formulas for the mixed canonical-grand-canonical partition function in Sec. II A [cf. Eqs. (7) and (8)] we immediately obtain the result

$$
\begin{gathered}
\left\langle a_{j}^{\dagger} a_{j}\right\rangle_{\mathrm{Q}^{0}}=\operatorname{dim}(\mathbf{Q}) \int_{\mathrm{SU}(N)} d \alpha_{1} d \alpha_{2} \cdots d \alpha_{r} M(\alpha) \chi_{\mathrm{Q}^{*}}^{*}(\alpha) \\
\times \operatorname{Tr}\left[\hat{\rho}_{0}^{\mu} \hat{\gamma}(\alpha) \hat{a}_{j}^{\dagger} \hat{a}_{j}\right],
\end{gathered}
$$

i.e., we find a group integral representation effecting the projection of a modified grand-canonical ensemble average [corresponding to the generating function $\widetilde{Z}$ introduced in Sec. II A, cf. Eq. (8)] onto a canonical one, which is specified by a definite $\mathrm{SU}(N)$ multiplet $\mathrm{Q}$; insertion of the definitions of $\hat{\gamma}$ and $\hat{\rho}_{0}^{\mu}$, the second of Eqs. (9) and (15), respectively, yields 


$$
\begin{aligned}
& \operatorname{Tr}\left[\hat{\rho}_{0}^{\mu} \hat{\gamma}(\boldsymbol{\alpha}) \hat{a}_{j}^{\dagger} \hat{a}_{j}\right] \\
& \quad=\operatorname{Tr}\left[\exp \left[-\beta \hat{H}_{0}(\boldsymbol{\mu})+i \sum_{\mu=1}^{r} \alpha_{\mu} \hat{Q}_{\mu}\right] \hat{a}_{j}^{\dagger} \hat{a}_{j}\right] .
\end{aligned}
$$

The rhs of Eq. (37) is a useful expression again involving at most only linear combinations of number operators in the exponential function and can be easily evaluated in practice [cf. the comment following Eq. (8) in Sec. II A].

Equations (34), which are well known, ${ }^{22}$ and Eqs. (35) - (37) show in detail the calculability of free singleparticle $g$-Green's functions and of $m$-Green's functions as well. Having these results in mind we finally return to the discussion of the perturbation expansion of $m$-Green's functions $G_{\mathrm{Q}}$, Eq. (28), for an interacting many-particle system.

First of all we incorporate the almost trivial modifications of the formalism due to passing from creation and destruction operators back to field operators by performing summations as required by Eq. (30b). Thus we replace creation and destruction operators by the corresponding field operators and quantum numbers $j, j^{\prime}$ by space variables $x, x^{\prime}$ in all relevant equations of this section from Eq. (23) on; e.g.,

$$
\begin{aligned}
G_{(\mathbf{Q})}\left(x, x^{\prime} ; \tau\right. & \left.-\tau^{\prime}\right) \\
& \equiv \sum_{j, j^{\prime}} G_{(\mathbf{Q})}\left(j, j^{\prime} ; \tau-\tau^{\prime}\right) \phi_{j}(x) \phi_{j^{\prime}}^{*}\left(x^{\prime}\right) \\
& =-Z_{(\mathbf{Q})}^{-1} \underset{(\mathbf{Q})}{\operatorname{Tr}}\left(\hat{\rho}^{\mu} T_{\tau}\left\{[\hat{\psi}(x, \tau)]_{H}\left[\hat{\psi}^{\dagger}\left(x^{\prime}, \tau^{\prime}\right)\right]_{H}\right\}\right) .
\end{aligned}
$$

Then, considering a rather general interaction $\hat{H}_{1}$ as discussed in connection with Eq. (30a), which is rewritten in the interaction picture,

$\hat{H}_{1}(\tau)=\int d^{3} x \hat{\psi}^{\dagger}(x, \tau)\left(e^{\tau \hat{H}_{0}(\boldsymbol{\mu})} \hat{h}_{1} e^{-\tau \hat{H}_{0}(\boldsymbol{\mu})}\right) \hat{\psi}(x, \tau)$,

we are able to draw several important conclusions from our analysis involving Wick's theorem at finite temperature respecting internal symmetry, Eq. (32), and the relations found between $g$ - and $m$-contractions and the respective types of Green's functions, Eqs. (33) [generalizations for field operators can immediately be written down with the help of Eqs. (30b) and (38)]: A general term of the perturbation expansion in the numerator or denominator on the rhs of Eq. (28) can be evaluated as a sum of products of free Green's functions corresponding to the sum of all possible fully contracted terms, where all internal variables $x_{i}, \tau_{i}\left(\neq x, \tau\right.$ or $x^{\prime}, t^{\prime}$ in case of the numerator) are integrated over space and "time" on account of Eqs. (28) and (39); of course, we are presently and always from now on talking about the field operator form of Eq. (28) where both sides are summed over all $j, j^{\prime}$ after multiplication with $\phi_{j}(x), \phi_{j^{\prime}}^{*}\left(x^{\prime}\right)$ in accordance with Eq. (38). Furthermore, each fully contracted term consists of exactly one m-Green's function multiplying a welldefined product of $\mathrm{g}$-Green's functions, where the fixed total number of factors at $n$th order and particularly the weight given to an individual contribution depend on the precise definition of the interaction Hamiltonian and will lead to the formulation of Feynman rules in practice. Anticipating an analysis of the various contributions to the rhs of Eq. (28) in terms of Feynman graphs, we distinguish disconnected from connected contributions. By definition in a disconnected contribution a sequel of internal space and time variables, which appear as arguments of Green's functions, and are completely integrated over space and time, closes on itself, whereas for a connected contribution the arguments interlace and finally are connected to the external reference variables $x, \tau$ and $x^{\prime}, \tau^{\prime}$. Omitting the integrations over internal variables and all details related to the "interaction bracket" [i.e., factors arising within or from terms in the parentheses in Eq. (39)] we can write, e.g., some typical products indicating various connected and disconnected parts [for $\hat{h}_{1}$ representing an external potential, cf. Eq. (30a)]:

$$
\begin{aligned}
& {\left[G^{0}\left(x_{1}, x_{2} ; \tau_{1}-\tau_{2}\right) G^{0}\left(x_{2}, x_{1} ; \tau_{2}-\tau_{1}\right)\right]_{\mathrm{disconn}}\left[G_{\mathrm{Q}}^{0}\left(x, x_{3} ; \tau-\tau_{3}\right) G^{0}\left(x_{3}, x^{\prime} ; \tau_{3}-\tau^{\prime}\right)\right]_{\mathrm{conn}},} \\
& {\left[G_{\mathrm{Q}}^{0}\left(x, x_{2} ; \tau-\tau_{2}\right) G^{0}\left(x_{1}, x_{2} ; \tau_{1}-\tau_{2}\right) G^{0}\left(x_{1}, x^{\prime} ; \tau_{1}-\tau^{\prime}\right)\right]_{\mathrm{conn}}} \\
& {\left[G^{0}\left(x_{1}, x_{2} ; \tau_{1}-\tau_{2}\right) G_{\mathrm{Q}}^{0}\left(x_{2}, x_{3} ; \tau_{2}-\tau_{3}\right) G^{0}\left(x_{3}, x_{1} ; \tau_{3}-\tau_{1}\right)\right]_{\mathrm{disconn}} .}
\end{aligned}
$$

We observe that the numerator on the rhs of Eq. (28) eventually will give rise to connected parts as well as products of Green's functions (diagrams) having a connected part together with a disconnected one, which factors. However, the denominator will produce disconnected diagrams only, since there are no external reference variables (points) $x, \tau$ and $x^{\prime}, \tau^{\prime}$. In the usual grand-canonical ensemble case it is straightforward to prove that all diagrams arising from the denominator in an analogous expansion, which can be "derived" from Eq. (28) by simply dropping the symbols $\mathbf{Q}$ everywhere, exactly cancel the disconnected parts occurring in the numerator; in this way one arrives at the famous connected-graphs expansion of single-particle g-Green's functions. ${ }^{22}$ In the present case of $m$-Green's functions the situation is more complicated, since we always have one $m$-Green's function $G_{\mathrm{Q}}^{0}$ among $g$-Green's functions $G^{0}$ [cf. examples in (40)]. $G_{\mathrm{Q}}^{0}$ is always found in a disconnected diagram occurring in the expansion of the denominator, whereas it can be found either in a disconnected or in a connected part of a diagram contributing to the numerator. Therefore, following the combinatorial consideration given in Ref. 22 for ordinary $g$-Green's functions, we obtain a different (graphical) characterization of the perturbation expansion of $m$-Green's function $G_{\mathrm{Q}}$ [Eq. (38)]: 


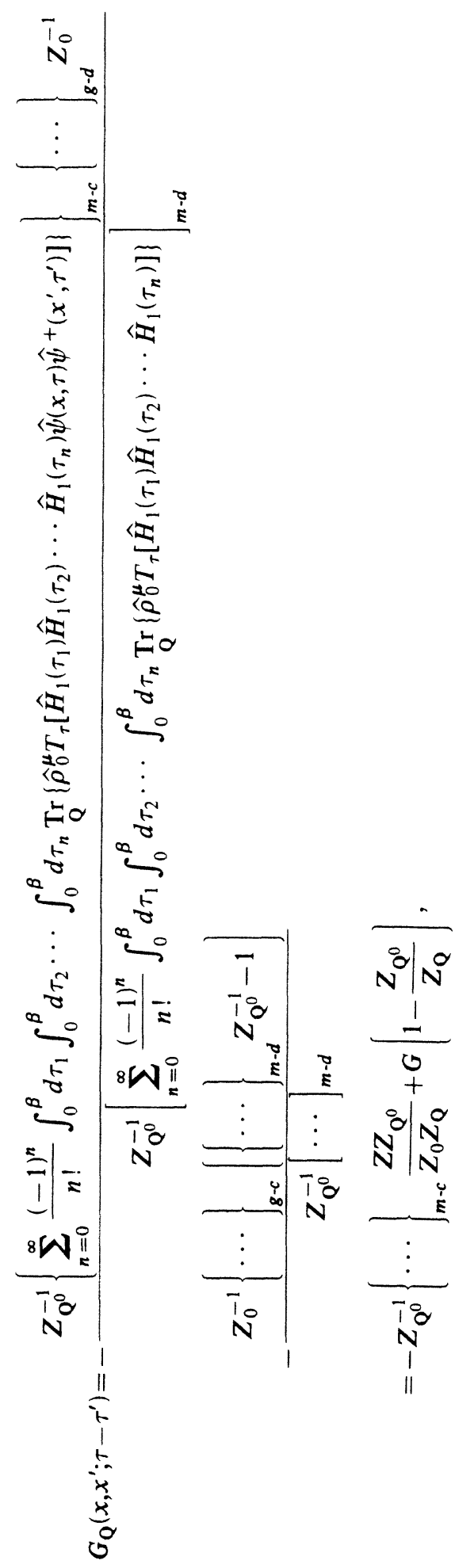

where $d$ and $c$ indicate that either disconnected or connected contributions, respectively, of the expressions in brackets have to be taken; the prefixes $m$ - and $g$ - distinguish the cases, where either the ordinary Wick's theorem ( $g$ for grand-canonical averaging) or the generalization given in Eq. (32) ( $m$ for mixed ensemble averaging) has to be applied when evaluating the terms in question; the subtraction in the second term of the numerator assures that the whole term does not contribute at zeroth order. The second equality in Eq. (41) has been obtained by resumming the denominator into $Z_{Q} / Z_{Q^{0}}$ [cf. Eqs. (27) and (28)] and realizing that the $g$ - $d$ part of the numerator equals the grand-canonical partition function $Z$ divided by its noninteracting limit $Z_{0}$, whereas the $g$ - $c$ part gives the full Green's function $-G$, cf. Eq. (38), for the grandcanonical ensemble. ${ }^{22}$ Thus in our approach we find no connected-graphs expansion for single-particle Green's functions $G_{\mathbf{Q}}$, Eq. (38), which is related to a mixed canonical-grand-canonical ensemble.

We conclude our study of single-particle Green's functions with the remark that the absence of a connectedgraphs expansion seems plausible in view of the fact that the canonical ensemble restriction to states of a definite $\mathrm{SU}(N)$ multiplet presents a global property. Therefore the propagation of a particle is not only influenced by other particles with which it interacts more or less directly, but also by the totality of charges present in the ensemble. This is in marked contrast with the situation in a grand-canonical ensemble where only the average local charge densities matter. To state it differently, in a canonical ensemble corresponding to some definite $\mathrm{SU}(N)$ representation $\mathbf{Q}$ there are no fluctuations with states belonging to a multiplet $\mathbf{Q}^{\prime}$. Since the result given in Eq. (41) shows a remarkably simple structure, it still may be useful in practical applications, if the partition functions $Z$ and $Z_{Q}$ are known perturbatively, which we already alluded to in the discussion following Eqs. (22) in Sec. II B. The absence of a connected-graphs expansion for a Green's function related to the mixed canonical-grandcanonical ensemble was also found in a different approach, which will be shortly mentioned in the following section, and a detailed example illustrating these results will be reported elsewhere.

\section{CONCLUSION}

In this paper we have formulated a general projection technique employing group-theoretical results to handle the description of systems by a quantum-statistical mixed canonical-grand-canonical ensemble. As was mentioned in the Introduction, this problem has quite an interesting history ${ }^{1-7}$ however, mostly the simplest $\mathrm{U}(1)$ internal symmetries only related to some exact charge conservation have been studied previously, with the exception of an early work by Bethe concerning the SU(2) group of spin. We also mention Ref. 24, where the SU(2) group of isospin conservation was studied recently with regard to $p \bar{p}$ annihilation.

Here we have presented a formalism which is general eno $y$ to deal with all compact semisimple Lie groups, although we restricted the more detailed description in 
Sec. II A to the case of an internal $\mathrm{SU}(N)$ group. Our main attention has been devoted to the investigation of interacting systems and we showed that well-known methods for the study of many-body systems, when suitably modified, can be applied here as well. Our results should help to extend the applicability of the projection technique beyond what was originally proposed in Ref. 1 .

A straightforward perturbation expansion for a mixed canonical-grand-canonical partition function has been developed in Sec. II B, which can be applied to the study of droplets of a weakly interacting quark-gluon plasma, which are intensely looked for in present and future highenergy experiments. ${ }^{13}$ Such a plasma droplet forms a rather well-defined finite system, where among other quantum numbers baryonic charge is conserved only on the average in a hot nuclear environment, but where color charges are still exactly confined to its interior, ${ }^{10-12,15}$ thus suggesting a statistical treatment involving a mixed canonical-grand-canonical ensemble. In Sec. III we studied the properties of suitably defined single-particle temperature Green's functions via a generalization of Wick's theorem respecting an exact internal symmetry. Our approach, however, did not produce an ordinary connectedgraphs expansion for the full Green's function, as is the case with grand-canonical ensemble considerations. ${ }^{22} \mathrm{We}$ argued that this could have been expected on physical reasons.

To further elucidate this point, we would like to mention that there exists at least one alternative approach to investigate single-particle Green's functions related to a mixed ensemble and we conclude with a technical remark.

One can start with the following definition of a generating Green's function:

$$
\begin{aligned}
& G\left(x, \tau ; x^{\prime}, \tau^{\prime} ; \boldsymbol{\alpha}\right) \\
& \quad \equiv-Z_{\mathbf{Q}}^{-1} \operatorname{Tr}\left(\hat{\rho}^{\mu} \hat{\gamma}(\alpha) T_{\tau}\left\{[\hat{\psi}(x, \tau)]_{H}\left[\hat{\psi}^{\dagger}\left(x,{ }^{\prime}, \tau^{\prime}\right)\right]_{H}\right\}\right),
\end{aligned}
$$

[cf. Eq. (38)], which is motivated by the successful study of a generating (partition) function in Sec. II B. But again one arrives at the conclusion that a connected-graphs expansion for the Green's function obtained from Eq. (42) by projecting onto the canonical ensemble is impossible, essentially because group integrals involved do not factor. Furthermore, the corresponding free generating Green's functions do not have the usual simple periodicity properties [cf. Eqs. (26)]. That is why we have chosen the approach presented in Sec. III of our paper.

\section{ACKNOWLEDGMENTS}

The authors wish to thank the Nuclear Theory Group very much for the kind hospitality extended to them and the inspiring atmosphere at Lawrence Berkeley Laboratory. H.-Th.E. gratefully acknowledges partial support by Deutscher Akademischer Austauschdienst and by North Atlantic Treaty Organization. Our work was supported by the Bundesministerium für Forschung und Technologie (Bonn, Germany) and by the U.S. Department of Energy under Contract No. DE-AC03-76SF00098.

\section{APPENDIX A: CHARGE OPERATORS AND CASIMIR OPERATORS}

We consider the (matrix) group $\mathrm{SU}(N)$ of special unitary transformations in $N$ dimensions. ${ }^{16-18}$ Its group elements can be parametrized by $n \equiv N^{2}-1$ real numbers; $n$ is called the order of the group. Correspondingly there are $n$ generators $\hat{g}_{\lambda}$ generating infinitesimally small transformations and which are related to the group elements $\hat{G} \in \mathrm{SU}(N)$ via

$$
\hat{G}\left(\alpha_{1}, \alpha_{2}, \ldots, \alpha_{n}\right)=\exp \left(i \sum_{\lambda=1}^{n} \alpha_{\lambda} \hat{g}_{\lambda}\right) .
$$

Unitarity implies that the generators are (linear) Hermitian operators characterized by real (additive) eigenvalues. There are $r \equiv N-1$ generators or linear combinations thereof which commute with each other; $r$ is called the rank of the group. We call these generators "charge operators" $\hat{Q}_{\mu}$, and their eigenvalues "charges," with

$$
\left[\hat{Q}_{\mu}, \hat{Q}_{\mu^{\prime}}\right]=0, \mu, \mu^{\prime}=1,2, \ldots, r .
$$

The Abelian subgroup generated by the charge operators is usually referred to as Cartan subgroup. Finally, there are $r$ nonlinear Casimir operators $\hat{C}_{v}$,

$$
\hat{C}_{v}=\sum_{\lambda=1}^{n} c_{\nu \lambda}\left(\hat{g}_{\lambda}\right)^{\nu+1}, v=1,2, \ldots, r
$$

which commute with all generators,

$$
\left[\widehat{C}_{v}, \hat{g}_{\lambda}\right]=0, v=1,2, \ldots, r, \lambda=1,2, \ldots, n \text {. }
$$

Therefore, the Casimir operators can be diagonalized together with all charge operators on a common basis set (vectors, tensors, etc.). The unitary irreducible representations among them or "multiplets" can be classified by an integer-valued $r$-dimensional vector with a one-to-one correspondence to eigenvalues of the $r$ Casimir operators.

\section{APPENDIX B: EXAMPLES OF GROUP CHARACTERS}

In this appendix we list some useful relations for characters of the groups $\mathrm{U}(1), \mathrm{SU}(2)$, and $\mathrm{SU}(3)$ which were derived in detail in Ref. 15. They may also serve as examples illustrating Eqs. (4)-(6) in Sec. II A.

(1) The unitary irreducible representations of $U(1)$ are given by complex phase factors $e^{i k \alpha}$ where $k$ is an integer and $\alpha(0 \leq \alpha<2 \pi)$ parametrizes the various group elements. The representations are one dimensional and the orthogonality relation for characters, Eq. (6), is identical to a well-known integral representation of the Kronecker $\delta$ :

$$
\int_{0}^{2 \pi} d \alpha \frac{1}{2 \pi} e^{-i k \alpha} e^{i k^{\prime} \alpha}=\delta_{k k^{\prime}}
$$

The measure function is a constant in this case, $M(\alpha)=1 / 2 \pi$.

(2) Unitary irreducible (vector) representations of $S U(2)$ are characterized by a non-negative integer $2 J$, i.e., $J$ is a non-negative half-integer (total spin quantum number). 
Their characters [see Eq. (4)] are real functions of a single variable $(0 \leq \alpha<4 \pi)$ :

$$
\chi_{J}(\alpha)=\sum_{J_{3}=-J}^{J} e^{i \alpha J_{3}}=\frac{\sin [(2 J+1) \alpha / 2]}{\sin (\alpha / 2)},
$$

where $J_{3}$ is an eigenvalue (magnetic quantum number) of the single charge operator belonging to $\mathrm{SU}(2)$ (cf. Appen$\operatorname{dix}$ A). From Eq. (B2) the dimensionality of representations is obtained according to Eq. (5):

$$
\operatorname{dim}(J)=\lim _{\alpha \rightarrow 0} \chi_{J}(\alpha)=2 J+1
$$

The orthogonality relation of characters becomes

$$
\int_{0}^{4 \pi} d \alpha \frac{1}{2 \pi} \sin ^{2}\left(\frac{\alpha}{2}\right) \chi_{J}(\alpha) \chi_{J^{\prime}}(\alpha)=\delta_{J J^{\prime}}
$$

from which the measure function $M(\alpha)$ $=(2 \pi)^{-1} \sin ^{2}(\alpha / 2)$ can be read off.

(3) For SU(3) unitary irreducible representations in terms of tensors were constructed in Refs. 15 and 19. They are specified by non-negative integers $P$ and $Q$, which in turn can be related to the eigenvalues of the two Casimir operators of SU(3) ${ }^{20}$ The characters are complex functions of two variables $(-\pi \leq \phi / 2, \phi / 3<\pi):{ }^{15}$

$$
\begin{aligned}
\chi_{P, Q}(\phi, \psi)= & \frac{\exp \left[\frac{2}{3} i(Q-P) \psi\right]}{4 \sin \left[\frac{1}{2}(\psi+\phi / 2)\right] \sin (\phi / 2) \sin \left[\frac{1}{2}(\psi-\phi / 2)\right]} \\
& \times\left[e^{i(P-Q) \psi} \sin \left[(P+Q+2) \frac{\phi}{2}\right]-e^{i(P+1) \psi} \sin \left[(P+1) \frac{\phi}{2}\right]-e^{-i(Q+1) \psi} \sin \left[(Q+1) \frac{\phi}{2}\right]\right] .
\end{aligned}
$$

The dimensionality formula is now calculated to be

$$
\operatorname{dim}(P, Q)=\lim _{\phi, \psi \rightarrow 0} \chi_{P, Q}(\phi, \psi)=\frac{1}{2}(P+1)(Q+1)(P+Q+2),
$$

and the following more complicated orthogonality relation is found:

$$
\frac{8}{3 \pi^{2}} \int_{-\pi}^{\pi} d\left(\frac{\phi}{2}\right) \int_{-\pi}^{\pi} d\left[\frac{\psi}{3}\right]\left\{\sin \left[\frac{1}{2}\left[\psi+\frac{\phi}{2}\right]\right] \sin \left[\frac{\phi}{2}\right] \sin \left[\frac{1}{2}\left[\psi-\frac{\phi}{2}\right)\right]\right\}^{2} \chi_{P, Q^{(}}^{*}(\phi, \psi) \chi_{P^{\prime}, Q^{\prime}}(\phi, \psi)=\delta_{P P^{\prime}} \delta_{Q Q^{\prime}} \text {. }
$$

The measure function thus is given by

$$
\begin{aligned}
M(\phi, \psi)=\frac{8}{3 \pi^{2}} & \left\{\sin \left[\frac{1}{2}\left[\psi+\frac{\phi}{2}\right]\right]\right. \\
& \left.\times \sin \left[\frac{\phi}{2}\right] \sin \left[\frac{1}{2}\left(\psi-\frac{\phi}{2}\right)\right]\right\}^{2} .
\end{aligned}
$$

\section{APPENDIX C: WICK'S THEOREM AT FINITE TEMPERATURE RESPECTING INTERNAL SYMMETRY}

To prove the generalized Wick's theorem, Eq. (32), stated in Sec. III it is necessary and sufficient to prove

$$
\begin{aligned}
& \left\langle T_{\tau}\left[\alpha_{a} \alpha_{b} \alpha_{c} \cdots \alpha_{m} \cdots \alpha_{n} \cdots \alpha_{x}\right]\right\rangle_{Q^{0}} \\
& =\alpha_{a} \alpha_{b} \alpha_{c} \cdots \alpha_{m}^{\tilde{m}} \cdots \alpha_{n}^{\sim} \cdots \alpha_{x} \\
& \quad+\alpha_{a} \alpha_{b} \alpha_{c} \cdots \alpha_{m}^{\sim} \cdots \alpha_{n}^{\sim} \cdots \alpha_{x}+\cdots
\end{aligned}
$$

(all possible fully contracted terms),

(C1)

where $\alpha_{j}$ denotes either a creation or a destruction operator in the interaction picture and the contractions were defined in Eqs. (31). The "time dependence" of these operators can be calculated from their equation of motion, e.g.,

$$
\begin{aligned}
\partial_{\tau} \hat{a}_{j}(\tau) & =e^{\tau \hat{H}_{0}(\boldsymbol{\mu})}\left[\hat{H}_{0}(\boldsymbol{\mu}), \hat{a}_{j}\right] e^{-\tau \hat{H}_{0}(\boldsymbol{\mu})} \\
& =-e_{j}(\boldsymbol{\mu}) \hat{a}_{j}(\tau)
\end{aligned}
$$

where we used Eq. (24) and respected Eq. (30c), which defines the diagonal representation of $\hat{H}_{0}(\boldsymbol{\mu})$. Equation $(\mathrm{C} 2)$ implies

$$
\hat{a}_{j}(\tau)=\hat{a}_{j} e^{-e_{j}(\boldsymbol{\mu}) \tau} .
$$

Similarly:

$$
\widehat{a}_{j}^{\dagger}(\tau)=\hat{a}_{j}^{\dagger} e^{+e_{j}(\boldsymbol{\mu}) \tau}
$$

or

$$
\alpha_{j}(\tau)=\alpha_{j}(0) e^{\lambda_{j} e_{j}(\mu) \tau},
$$

where $\lambda_{j}=+1(-1)$ for $\alpha_{j}$ designating a creation (destruction) operator. The next steps follow the proof of Wick's theorem for grand-canonical ensemble averages given in Ref. 22. It is sufficient to consider the special case where time ordering has already been performed in Eq. (C1), because otherwise reordering operators on both sides simultaneously does not introduce any additional changes of (relative) sign. From the left-hand side (lhs) of Eq. (C1) we then obtain, by commuting $\alpha_{a}$ successively to the right,

$$
\begin{aligned}
& \left\langle\alpha_{a} \alpha_{b} \alpha_{c} \cdots \alpha_{x}\right\rangle_{Q^{0}} \\
& =\left[\alpha_{a}, \alpha_{b}\right]_{ \pm}\left\langle\alpha_{c} \cdots \alpha_{x}\right\rangle_{Q^{0}} \\
& \quad \mp\left[\alpha_{a}, \alpha_{c}\right]_{ \pm}\left\langle\alpha_{b} \cdots \alpha_{x}\right\rangle_{Q^{0}}+\cdots \\
& \quad+\left[\alpha_{a}, \alpha_{x}\right]_{ \pm}\left\langle\alpha_{b} \alpha_{c} \cdots \alpha_{x}\right\rangle_{Q^{0}} \mp\left\langle\alpha_{b} \alpha_{c} \cdots \alpha_{x} \alpha_{a}\right\rangle_{Q^{0}}
\end{aligned}
$$


where we have taken the (anti)commutators referring to bosons (fermions) outside the traces-henceforth lower (upper) signs refer to bosons (fermions)-and we noticed the fact that the total number of $\alpha$ 's must be even; traces vanish in the noninteracting ensemble unless the number of creation operators equals the number of destruction operators. Using the cyclic property of the trace and Eq. (C3c), the last term in Eq. (C4) becomes

$$
\begin{aligned}
\mp Z_{\mathbf{Q}^{0}}^{-1} \operatorname{Tr}\left(\alpha_{a} \hat{\rho}_{b}^{\mu} \alpha_{b} \alpha_{c} \cdots \alpha_{x}\right) \\
=\mp e^{\lambda_{a} e_{a}(\mu) \beta} Z_{\mathbf{Q}^{0}}^{-1} \operatorname{Tr}\left(\hat{\rho}_{0}^{\mu} \alpha_{a} \alpha_{b} \alpha_{c} \cdots \alpha_{x}\right) .
\end{aligned}
$$

Therefore, collecting terms in Eq. (C4) together with Eq. (C5), we find

$$
\begin{aligned}
\left\langle\alpha_{a} \alpha_{b} \alpha_{c} \cdots \alpha_{x}\right\rangle_{\mathbf{Q}^{0}=} & \left(\alpha_{a} \alpha_{b}\right)\left\langle\alpha_{c} \cdots \alpha_{x}\right\rangle \\
& \mp\left(\alpha_{a} \alpha_{c}\right)\left\langle\alpha_{b} \cdots \alpha_{x}\right\rangle_{\mathbf{Q}^{0}}+\cdots \\
& +\left(\alpha_{a} \alpha_{x}\right)\left\langle\alpha_{b} \alpha_{c} \cdots \alpha_{x}\right\rangle_{\mathbf{Q}^{0}},
\end{aligned}
$$

with the abbreviation

$$
\left(\alpha_{i} \alpha_{j}\right) \equiv \frac{\left[\alpha_{i}, \alpha_{j}\right]_{ \pm}}{1 \pm e^{\lambda_{i} e_{i}(\boldsymbol{\mu}) \beta}} .
$$

These brackets, however, can be calculated by using the (anti)commutation rules and extracting the time dependence of operators by Eq. (C3c); the only nonvanishing ones are

$$
\begin{aligned}
\left(\hat{a}_{i}^{\dagger}(\tau) \widehat{a}_{i}\left(\tau^{\prime}\right)\right) & =\frac{1}{e^{\beta e_{i}(\boldsymbol{\mu})} \pm 1} e^{e_{i}(\boldsymbol{\mu})\left(\tau-\tau^{\prime}\right)} \\
& =\left\langle\hat{a}_{i}^{\dagger} \hat{a}_{i}\right\rangle_{0} e^{\boldsymbol{e}_{i}(\boldsymbol{\mu})\left(\tau-\tau^{\prime}\right)} \\
\left(\hat{a}_{i}(\tau) \widehat{a}_{i}^{\dagger}\left(\tau^{\prime}\right)\right) & =\frac{1}{1 \pm e^{-\beta e_{i}(\boldsymbol{\mu})} e^{-e_{i}(\boldsymbol{\mu})\left(\tau-\tau^{\prime}\right)}} \\
& =\left(1 \mp\left\langle\hat{a}_{i}^{\dagger} \widehat{a}_{i}\right\rangle_{0}\right) e^{-e_{i}(\boldsymbol{\mu})\left(\tau-\tau^{\prime}\right)} \\
& =\left\langle\hat{a}_{i} \hat{a}_{i}^{\dagger}\right)_{0} e^{-e_{i}(\boldsymbol{\mu})\left(\tau-\tau^{\prime}\right)}
\end{aligned}
$$

i.e., they can be simply expressed in terms of grandcanonical ensemble averages of number operators. Assuming $\tau_{a}>\tau_{b}$, Eqs. (C8) can be combined to yield

$$
\left(\alpha_{a} \alpha_{b}\right)=\left\langle T_{\tau}\left[\alpha_{a} \alpha_{b}\right]\right\rangle_{0}=\alpha_{a} \alpha_{b},
$$

where we used the definition of a g-contraction, Eq. (31b). We obtain from Eq. (C6) together with Eq. (C9):

$$
\begin{aligned}
\left\langle T_{\tau}\left[\alpha_{a} \alpha_{b} \alpha_{c} \cdots \alpha_{x}\right]\right\rangle_{\mathbf{Q}^{0}} & =\alpha_{a}^{\prime} \alpha_{b}\left\langle T_{\tau}\left[\alpha_{c} \cdots \alpha_{x}\right]\right\rangle_{\mathbf{Q}^{0}} \mp \alpha_{a} \alpha_{c}\left\langle T_{\tau}\left[\alpha_{b} \cdots \alpha_{x}\right]\right\rangle_{Q^{0}}+\cdots+\alpha_{a} \alpha_{x}\left\langle T_{\tau}\left[\alpha_{b} \alpha_{c} \cdots\right]\right\rangle_{Q^{0}} \\
& \equiv\left\langle T_{\tau}\left[\alpha_{a} \alpha_{b} \alpha_{c} \cdots \alpha_{x}\right]\right\rangle_{Q^{0}}+\left\langle T_{\tau}\left[\alpha_{a} \alpha_{b} \alpha_{c} \cdots \alpha_{x}\right]\right\rangle_{Q^{0}}+\cdots+\left\langle T_{\tau}\left[\alpha_{a} \alpha_{b} \alpha_{c} \cdots \alpha_{x}\right]\right\rangle_{Q^{0}},
\end{aligned}
$$

where we put in $T_{\tau}$ reminding the reader that we assumed $\tau_{a}>\tau_{b}>\tau_{c}>\cdots>\tau_{x}$ in Eq. (C4) and therefore in Eq. (C6) as well, and where the last equality holds as a definition.

Equation (C10) presents the important first step towards Wick's theorem. Since the contractions can be taken out of the traces on the rhs of Eq. (C10) being merely scalar factors, the same analysis can be applied over and over again until all possible pairs of operators are $g$ con- tracted except a last one; we have to consider

$$
\begin{aligned}
\left\langle T_{\tau}\left[\alpha_{i} \alpha_{j}\right]\right\rangle_{\mathbf{Q}^{0}} & \equiv Z_{\mathbf{Q}^{0}}^{-1} \operatorname{Tr}\left(\hat{\rho}_{0}^{\mu} T_{\tau}\left[\alpha_{i} \alpha_{j}\right]\right) \\
& \equiv \alpha_{i}^{\tilde{\alpha} \alpha_{j}},
\end{aligned}
$$

by definition [Eq. (31a)]; Eq. (C11) is further evaluated in Eqs. (33a) and (33b) in Sec. III. This remark completes the proof of Eq. (C1).
${ }^{1}$ K. Redlich and L. Turko, Z. Phys. C 5, 201 (1980); L. Turko, Phys. Lett. 104B, 153 (1981).

${ }^{2}$ H. A. Bethe, Phys. Rev. 50, 332 (1936).

3J. V. Lepore and R. N. Stuart, Phys. Rev. 94, 1724 (1954); V. B. Magalinskii and I. P. Terletskii, Zh. Eksp. Teor. Fiz. 32, 584 (1957) [Sov. Phys.-JETP 5, 483 (1957)].

${ }^{4}$ V. B. Magalinskii and I. P. Terletskii, Zh. Eksp. Teor. Fiz. 29, 151 (1955) [Sov. Phys._JETP 2, 143 (1956)].

5J. Rafelski, M. Danos, Phys. Lett. 97B, 279 (1980).

${ }^{6}$ C. Derreth, W. Greiner, H.-Th. Elze, and J. Rafelski, Phys. Rev. C 31, 1360 (1985).

7J. Kapusta, Nucl. Phys. B196, 1 (1982).

${ }^{8}$ C. N. Yang and R. Mills, Phys. Rev. 96, 191 (1954).

${ }^{9}$ E. S. Abers and B. V. Lee, Phys. Rev. 9, 1 (1973).

${ }^{10}$ H.-Th. Elze, J. Rafelski, and W. Greiner, Verh. Dtsch. Phys. Ges. 9/1980, 1226 (1980); 6/1982, 1285 (1982); Gesellschaft für Schwerionenforschung Darmstadt Report 83-1 (1983) 132; H.-Th. Elze, W. Greiner, P. Koch, B. Müller, and J. Rafelski, Verh. Dtsch. Phys. Ges. 6/1983, 1191 (1983).

${ }^{11}$ H.-Th. Elze, W. Greiner, and J. Rafelski, Phys. Lett. 124B, 515 (1983); Gesellschaft für Schwerionenforschung Darmstadt Report 84-1 (1984) 144; Z. Phys. C 24, 361 (1984).

${ }_{12}$ M. I. Gorenstein, S. I. Lipshikh, V. K. Petrov, and G. M. Zinovjev, Phys. Lett. 123B, 437 (1983).

${ }^{13}$ The following collection of reports and conference proceedings may guide the reader to the numerous quark-gluon plasma papers: Thermodynamics of Quarks and Hadrons, edited by H. Satz (North-Holland, Amsterdam, 1981); Quark Matter Formation and Heavy Ion Collisions, edited by M. Jacob and H. Satz (World Scientific, Singapore, 1982); Quark Matter Formation and Heavy Ion Collisions, edited by M. Jacob and J. Tran Thanh Van [Phys. Rep. 88, 321 (1982)]; Quark Matter 
'83, edited by T. W. Ludlam and H. E. Wegner [Nucl. Phys. A418 (1984)]; B. Müller, The Physics of the Quark-Gluon Plasma, Vol. 225 of Springer Lecture Notes in Physics (Springer, Berlin, 1985).

${ }^{14}$ W. Marciano and H. Pagels, Phys. Rep. 36, 137 (1978); E. V. Shuryak, ibid. 115, 151 (1984).

${ }^{15}$ H.-Th. Elze, Ph.D. thesis, Frankfurt am Main, 1985 (unpublished).

${ }^{16} \mathrm{H}$. Weyl, The Classical Groups (Princeton University, Princeton, 1946).

${ }^{17} \mathrm{~W}$. Miller, Symmetry Groups and Their Applications, (Academic, New York, 1972).

${ }^{18}$ W. Greiner, Theoretische Physik: Quantenmechanik II-
Symmetrien (Deutsch, Thun und Frankfurt am Main, 1981).

${ }^{19}$ J. McL. Emmerson, Symmetry Principles in Particle Physics (Clarendon, Oxford, 1972).

${ }^{20}$ A. Th. M. Aerts, P. J. G. Mulders, and J. J. de Swart, Phys. Rev. D 17, 260 (1978).

${ }^{21}$ R. P. Feynman, Statistical Mechanics (Benjamin, Reading, Mass., 1972).

${ }^{22}$ A. L. Fetter and J. D. Walecka, Quantum Theory of ManyParticle Systems (McGraw-Hill, New York, 1971).

${ }^{23}$ R. Kubo, M. Toda, and N. Hashitsume, Statistical Physics II (Springer-Verlag, Berlin, 1985).

${ }^{24}$ B. Mueller and J. Rafelski, Phys. Lett. 116B, 274 (1982). 